\title{
COMBINING CLAYS AND ULTRASOUND IRRADIATION FOR AN O-ACETYLATION REACTION OF $N$-GLUCOPYRANOSYL AND OTHER MOLECULES
}

\author{
RONALDO N. DE OLIVEIRA, * AUGUSTO DE L. XAVIER, BRUNA M. GUIMARÃES, VALENTINA N. \\ E MELO, WAGNER O. VALENÇA, WILSON S. DO NASCIMENTO, POLLYANNA L. F. DA COSTA AND \\ CELSO A. CAMARA
}

\author{
Departamento de Ciências Moleculares, Laboratório de Sintese de Compostos Bioativos, Universidade Federal Rural de Pernambuco, \\ Rua Dom Manoel de Medeiros S/N, Dois Irmãos, 52171-900, Recife, PE, Brazil.
}

\begin{abstract}
A convenient, efficient and fast acetylation combining clays and ultrasound irradiation is described. Some molecules from nature or synthetic source, i.e., D-glucose, glycerol, D-mannitol and 1,2,3-triazolic derivatives such as $N$-glucosyl sugars and 2-substituted 1,4-naphthoquinone structures were acetylated. This kind of chemistry may be classified as eco-friendly because the reactions take a short time and the catalyst is reusable.
\end{abstract}

Keywords: montmorillonites, carbohydrate, glycerol, acetylation, ultrasound.

\section{INTRODUCTION}

The development of chemical building blocks inspired by nature is commonly reported in scientific articles. ${ }^{1}$ Many natural and synthetic products have had their structures modified by chemical procedures. carboxylic esters are considered very important functional groups in natural products and as intermediates for organic synthesis. The pharmacological properties of some molecules can be modified by a simple reaction of acetylation, a specific case being the acetylation of salicylic acid (SA) to furnish acetyl salicylic acid (ASA). In this context, one of the most used reactions is the acetylation of alcohols. Recent works described various acetylating systems, for example with $\mathrm{Ph}, \mathrm{P}(\mathrm{OAc})_{2},{ }^{2}$ triethyl orthoacetate, ${ }^{3}$ vinyl acetate- $\mathrm{I}_{2},{ }^{4}$ acetyl chloride or $\mathrm{Ac}_{2} \mathrm{O}$-DMAP-py. ${ }^{5}$ In addition, clays are frequently employed as heterogeneous systems to promote acetylation reaction. ${ }^{6,7}$ Specifically, the acylation of alcohols has been carried out using montmorillonite K10 impregnated with some metals, such as $\mathrm{FeCl}_{3},{ }^{6} \mathrm{InCl}_{3},{ }^{8}$, and $\mathrm{Cu}^{2+}, 9$ among others. ${ }^{10}$

Introduction of new laboratory techniques is noteworthy exciting for the undergraduate students involved in organic chemistry experiments. One fast-growing area is organic synthesis using an ultrasound irradiation bath. ${ }^{11}$ Our research group has been using the ultrasonic technique for the synthesis of amidoximes, ${ }^{12}$ carbohydrates ${ }^{13}$ and 1,2,3-triazole derivatives via click chemistry. ${ }^{14}$ However, there are only a few examples reported in the literature that combine clays and ultrasound energy ${ }^{15}$ and only one example for acetylation using this technique..$^{16}$ In general, this kind of chemistry is considered ecofriendly because these procedures are known for their efficiency, short reaction time and easily removable and reusable catalyst.

After started our studies ${ }^{17}$ we decided to prepare a diversity of acetylated molecules of natural or synthetic origin using clays (montmorillonite $\mathrm{K} 10$ or $\mathrm{KSF}$ ), acetic anhydride and ultrasonic energy.

\section{EXPERIMENTAL}

\section{General}

All organic solvents were analytical grade (Vetec-Brazil). All reactions were monitored by TLC analysis containing GF-254 (Merck-Darmstadt, Germany). The clays (montmorillonite K10 and KSF) were purchased from Aldrich. Reactions were carried out in a USC-1400A Ultracleaner ultrasound cleaning bath with an operating frequency of $40 \mathrm{kHz}$. Column chromatography was performed on Merck silica gel 60 , optical rotations were measured using an Anton-Paar MCP 200 polarimeter and values are given in deg. $\mathrm{cm}^{2}, \mathrm{~g}^{-1}$; Melting points were determined in a PFM II BioSan apparatus and are uncorrected. ${ }^{1} \mathrm{H}$ $(400 \mathrm{MHz}),{ }^{13} \mathrm{C}$ NMR $(100 \mathrm{MHz})$ spectra were obtained in Varian Unity Plus at 400 spectrometers in $\mathrm{CDCl}_{3}$. Elemental analyses were carried out in a $\mathrm{CA}$ EA1110 CHNS-O analyzer. GC-MS $\left(60-240{ }^{\circ} \mathrm{C}\right.$ at $3{ }^{\circ} \mathrm{C}$ min rates) on a Varian 431-GC device coupled to a Varian 220-MS instrument using a fused-silica capillary column $(30 \mathrm{~m} \times 0.25 \mathrm{~mm}$ i.d. $\times 0.25 \mu \mathrm{m})$ coated with DB-5. MS spectra were obtained using EI mode at $70 \mathrm{eV}$, at scan intervals of $0.5 \mathrm{~s}$ and fragments from 40 to $550 \mathrm{Da}$. The compounds $\mathbf{1}$ and $\mathbf{2}$ were synthesized using literature procedure, and their analyses are comparable with reported data. ${ }^{13}$ The compounds $\mathbf{5 a}, \mathbf{5 b}$ and 2-amino-1,4-naphthoquinone are comparable with reported data. ${ }^{18}$

Typical experimental procedure for synthesis of $\mathrm{N}$-glycosides (3a-d)

We employed the methodology adapted from literature. ${ }^{13}$ We only used acetonitrile instead of the dichloromethane solvent.

1'-(2,3,4,6-Tetra- $O$-acetyl- $\beta$-D-glucopyranosyl)-4'-(3-hydroxypropyl)$1 H_{-1}$ ', 2',3'-triazole (3a)

Solid; mp 160-162 ${ }^{\circ} \mathrm{C}$; $[\mathrm{a}]{ }_{\mathrm{D}}{ }^{25}=50.0\left(\mathrm{C}=0.01 / \mathrm{CH}_{2} \mathrm{CL}_{2}\right) ;{ }^{1} \mathrm{H}$ NMR $(400 \mathrm{MHz}$, $\left.\mathrm{CDCl}_{3}\right): \delta 7.68\left(\mathrm{br} \mathrm{s}, 1 \mathrm{H}, \mathrm{H}_{\text {triaz }}\right), 5.81(\mathrm{~d}, 1 \mathrm{H}, J 7.6 \mathrm{~Hz}, \mathrm{H}-1), 5.39-5.31(\mathrm{~m}, 3 \mathrm{H}$, $\mathrm{H}-2, \mathrm{H}-3, \mathrm{H}-4), 4.29-4.21$ (m, $1 \mathrm{H}, \mathrm{H}-6 \mathrm{a}), 4.10$ (br d, $1 \mathrm{H}, J 12.0 \mathrm{~Hz} \mathrm{H}-6 \mathrm{~b}), 3.98$ (m, 1H, H-5), 3.65 (t, 2H, $\mathrm{CH}_{2}$ ), 3.07 (t, 2H, $\left.\mathrm{CH}_{2}\right), 2.85$ (t, 2H, $\left.\mathrm{CH}_{2}\right), 2.02(\mathrm{~s}$, $\left.3 \mathrm{H}, \mathrm{CH}_{3} \mathrm{CO}\right), 2.00\left(\mathrm{~s}, 3 \mathrm{H}, \mathrm{CH}_{3} \mathrm{CO}\right), 1.96\left(\mathrm{~s}, 3 \mathrm{H}, \mathrm{CH}_{3} \mathrm{CO}\right), 1.82\left(\mathrm{~s}, 3 \mathrm{H}, \mathrm{CH}_{3} \mathrm{CO}\right)$. ${ }^{13} \mathrm{C}$ NMR $\left(100 \mathrm{MHz}, \mathrm{CDCl}_{3}\right): \delta 170.5,169.3,169.1,169.0,128.0,110.0,86.3$, $75.3,70.8,72.5,70.4,67.6,61.5,31.5,27.9,21.7,20.7,20.5,20.2,20.1$. Anal. Calc.: $\mathrm{C}_{19} \mathrm{H}_{27} \mathrm{~N}_{3} \mathrm{O}_{10}: \mathrm{C}, 49.89 ; \mathrm{H}, 5.95$. Found: $\mathrm{C}, 50.02 ; \mathrm{H}, 5.89$.

$( \pm)-1$ ' $-(2,3,4,6$-Tetra- $O$-acetyl- $\beta$-D-glucopyranosyl $)-4^{\prime}-(2$ hydroxypropyl)-1H-1',2',3'-triazole (3b)

Solid; mp $170-172{ }^{\circ} \mathrm{C} ;[\mathrm{a}]_{\mathrm{D}}{ }^{25}=-80.0\left(\mathrm{C}=0.01 / \mathrm{CH}_{2} \mathrm{CL}_{2}\right) ;{ }^{1} \mathrm{H}$ NMR $(400$ $\left.\mathrm{MHz}, \mathrm{CDCl}_{3}\right): \delta 7.75(\mathrm{br} \mathrm{s}, 1 \mathrm{H}, \mathrm{H}$ ), $5.87($ br s, $1 \mathrm{H}, \mathrm{H}-1), 5.46-5.22(\mathrm{~m}, 3 \mathrm{H}$, $\mathrm{H}-2, \mathrm{H}-3, \mathrm{H}-4), 4.34-4.15$ (m, 3H, H-6a, H-6b, CHO), 4.09 (m, 1H, H-5), 2.93$2.76\left(\mathrm{~m}, 3 \mathrm{H}, \mathrm{OH}, \mathrm{CH}_{2}\right), 2.09\left(\mathrm{~s}, 3 \mathrm{H}, \mathrm{CH}_{3} \mathrm{CO}\right), 2.07\left(\mathrm{~s}, 3 \mathrm{H}, \mathrm{CH}_{3} \mathrm{CO}\right), 2.03(\mathrm{~s}, 3 \mathrm{H}$, $\left.\mathrm{CH}_{3} \mathrm{CO}\right), 1.88\left(\mathrm{~s}, 3 \mathrm{H}, \mathrm{CH}_{3} \mathrm{CO}\right) .{ }^{13} \mathrm{C}$ NMR $\left(100 \mathrm{MHz}, \mathrm{CDCl}_{3}\right): \delta 170.5,169.9$, $169.3,169.0,120.6,144.7,86.3,75.3,72.3,70.7,67.7,61.5,34.6,34.4,22.9$, 20.7, 20.5, 20.4, 20.2. Anal. Calc.: $\mathrm{C}_{19} \mathrm{H}_{27} \mathrm{~N}_{3} \mathrm{O}_{10}$ : C, 49.89; H, 5.95. Found: $\mathrm{C}$, $49.66 ; \mathrm{H}, 6.05$

( \pm )-1 ' - $(2,3,4,6$-Tetra- $O$-acetyl- $\beta$-D-glucopyranosyl $)-4$ ' - (1hydroxyethyl)-1H-1',2',3'-triazole (3c)

Solid; mp 193-195 ${ }^{\circ} \mathrm{C}$; [a] ${ }^{25}=-16.5\left(\mathrm{C}=0.01 / \mathrm{CH}_{2} \mathrm{CL}_{2}\right) ;{ }^{1} \mathrm{H}$ NMR $(400$ $\left.\mathrm{MHz}, \mathrm{CDCl}_{3}\right): 7.73\left(\mathrm{~s}, 1 \mathrm{H}, \mathrm{H}_{\mathrm{triaz}}\right), 5.87\left(\mathrm{~d}, 1 \mathrm{H}, J 8.8 \mathrm{~Hz}, \mathrm{H}^{-1}\right), 5.43-5.24(\mathrm{~m}, 3 \mathrm{H}$, $\mathrm{H}-2, \mathrm{H}-3, \mathrm{H}-4), 5.07$ (q, $J 6.0 \mathrm{~Hz}, \mathrm{CHO}), 4.30(\mathrm{dd}, 1 \mathrm{H}, J 5.2,12.4 \mathrm{~Hz}, \mathrm{H}-6$ ), 4.15 (br d, $1 \mathrm{H}, J 12.4 \mathrm{~Hz}, \mathrm{H}-6$ ) $, 4.01(\mathrm{~m}, 1 \mathrm{H}, \mathrm{H}-5), 2.08$ (s, 3H, $\left.\mathrm{CH}_{3} \mathrm{CO}\right), 2.07$ (s, $\left.3 \mathrm{H}, \mathrm{CH}_{3} \mathrm{CO}\right), 2.03\left(\mathrm{~s}, 3 \mathrm{H}, \mathrm{CH}_{3} \mathrm{CO}\right), 1.88\left(\mathrm{~s}, 3 \mathrm{H}, \mathrm{CH}_{3} \mathrm{CO}\right), 1.60$ (br d, $3 \mathrm{H}, J$ $\left.6.8 \mathrm{~Hz}, \mathrm{CH}_{3}\right) .{ }^{13} \mathrm{C}$ NMR $\left(100 \mathrm{MHz}^{3} \mathrm{CDCl}_{3}\right): \delta 170.5,169.9,169.3,168.9,153.1$, 118.6, 85.7, 75.1, 72.6, 70.3, 67.7, 63.1, 63.0, 61.5, 23.0, 22.9, 20.6, 20.5, 20.1. Anal. Calc.: $\mathrm{C}_{18} \mathrm{H}_{25} \mathrm{~N}_{3} \mathrm{O}_{10}$ : C, 48.76; H, 5.68. Found: $\mathrm{C}, 48.50 ; \mathrm{H}, 6.01$.

$1^{\prime}$ ' $(2,3,4,6$ - Tetra-O-acetyl- $\beta$-D - glucopyranosyl $)-4$ ' - (1 hydroxycyclohexyl)-1H-1',2',3'-triazole (3d)

Solid; mp $175-179^{\circ} \mathrm{C}$; [a] ${ }_{\mathrm{D}}^{25}=-3.8\left(\mathrm{C}=0.01 / \mathrm{CH}_{2} \mathrm{CL}_{2}\right){ }^{1} \mathrm{H}$ NMR $(400 \mathrm{MHz}$, $\left.\mathrm{CDCl}_{3}\right): 7.70\left(\mathrm{~s}, 1 \mathrm{H}, \mathrm{H}_{\mathrm{H}}\right), 5.85(\mathrm{~d}, 1 \mathrm{H}, J 8.8 \mathrm{~Hz}, \mathrm{H}-1), 5.43,5.23(\mathrm{~m}, 3 \mathrm{H}, \mathrm{H}-2$, H-3, H-4), 4.30 (dd, 1 H, J J 5.2, $12.4 \mathrm{~Hz}, \mathrm{H}-6 \mathrm{a}), 4.15$ (br d, $1 \mathrm{H}, J 12.4 \mathrm{~Hz}, \mathrm{H}-6 \mathrm{~b}$ ), $4.00(\mathrm{~m}, 1 \mathrm{H}, \mathrm{H}-5), 2.08\left(\mathrm{~s}, 3 \mathrm{H}, \mathrm{CH}_{3} \mathrm{CO}\right), 2.06(\mathrm{~s}, 3 \mathrm{H}, \mathrm{CH}, \mathrm{CO}), 2.02(\mathrm{~s}, 3 \mathrm{H}$, $\left.\mathrm{CH}_{3} \mathrm{CO}\right), 1.85$ (s, $\left.3 \mathrm{H}, \mathrm{CH}_{3} \mathrm{CO}\right), 1.80-1.25$ (m, 10H, cyclohexyl). ${ }^{13} \mathrm{C}$ NMR $(100$ $\left.\mathrm{MHz}, \mathrm{CDCl}_{3}\right): \delta 170.5,169.9,169.3,168.8,145.1,118.2,85.8,75.1,72.6,70.3$, 
69.5, 67.8, 61.6, 38.0, 25.3, 21.9, 20.5, 20.1 Anal. Calc.: $\mathrm{C}_{22} \mathrm{H}_{31} \mathrm{~N}_{3} \mathrm{O}_{10}$ : C, 53.11; H, 6.28. Found: C, 52.83; H, 6.46 .

Typical experimental procedure for synthesis of acetylated compounds An intimate mixture of hydroxyl compounds $(1 \mathrm{mmol})$ and clay $(200$ $\%$ weight/substrate) were well mixed individually and placed in a $125 \mathrm{~mL}$ erlenmeyer followed by the addition of acetic anhydride ( 4 equiv/OH). The mixture was irradiated in an ultrasonic bath at the indicated temperature for a specific time. The completion of the reaction was verified by use of thin-layer chromatography (TLC). After this, $10 \mathrm{~mL}$ de ethyl acetate was added and the crude material was filtered and the resulting organic phase was washed with water and a $\mathrm{NaHCO}_{3}$ saturated solution and dried with anhydrous sodium sulfate. Then, column chromatography with gradient ethyl acetate $/ n$-hexane was used to generate the desired pure products. We have tested to scale-up the present method at $25 \mathrm{mmol}$ scale to obtain comparable results for the compounds $\mathbf{1}$ and 10.

1 '-(2,3,4,6-Tetra- $O$-acetyl- $\beta$-D-glucopyranosyl)-4'-(3-O-acetyl-propyl)$1 H-1$ ',2',3'-triazole (4a)

Solid; mp $143-145^{\circ} \mathrm{C}$; [a] ${ }_{\mathrm{D}}{ }^{25}=-40.0\left(\mathrm{C}=0.01 / \mathrm{CH}_{2} \mathrm{CL}_{2}\right) ;{ }^{1} \mathrm{H}$ NMR $(400 \mathrm{MHz}$, $\left.\mathrm{CDCl}_{3}\right): \delta 7.56\left(\mathrm{~s}, 1 \mathrm{H}, \mathrm{H}_{\text {triaz }}\right), 5.85(\mathrm{~d}, 1 \mathrm{H}, J 9.0 \mathrm{~Hz}, \mathrm{H}-1), 5.42-5.23(\mathrm{~m}, 3 \mathrm{H}, \mathrm{H}-2$, $\mathrm{H}-3, \mathrm{H}-4), 4.30$ (dd, $1 \mathrm{H}, J 12.5,7.4 \mathrm{~Hz}, \mathrm{H}-6 \mathrm{a}), 4.14$ (br d, $1 \mathrm{H}, J 12.5 \mathrm{~Hz}, \mathrm{H}-6 \mathrm{~b}$ ), $4.10\left(\mathrm{t}, 2 \mathrm{H}, J 6.3 \mathrm{~Hz}, \mathrm{CH}_{2} \mathrm{O}\right), 4.01-3.98(\mathrm{br} \mathrm{dd}, 1 \mathrm{H}, 1 \mathrm{H}, J 10.1,3.0 \mathrm{~Hz}, \mathrm{H}-5), 2.81$ $\left(\mathrm{t}, 2 \mathrm{H}, J 7.5 \mathrm{~Hz}, \mathrm{CH}_{2}\right), 2.08\left(\mathrm{~s}, 3 \mathrm{H}, \mathrm{CH}_{3} \mathrm{CO}\right), 2.06\left(\mathrm{~s}, 3 \mathrm{H}, \mathrm{CH}_{3} \mathrm{CO}\right), 2.05(\mathrm{~s}, 3 \mathrm{H}$, $\left.\mathrm{CH}_{3} \mathrm{CO}\right), 2.02-199\left(\mathrm{~m}, 5 \mathrm{H}, \mathrm{CH}_{2}, \mathrm{CH}_{3} \mathrm{CO}\right), 1.87$ (s, 3H, $\left.\mathrm{CH}_{3} \mathrm{CO}\right) .{ }^{3} \mathrm{C}$ NMR $(100$ $\left.\mathrm{MHz}^{3} \mathrm{CDCl}_{3}\right): \delta 171.1,170.5,169.9,169.4,169.0,147.7,119.2,85.7,75.1,72.6$, 70.2, 67.7, 63.3, 61.5, 28.0, 22.4, 22.1, 20.7, 20.1. Anal. Calc.: $\mathrm{C}_{21} \mathrm{H}_{29} \mathrm{~N}_{3} \mathrm{O}_{11}: \mathrm{C}$, $50.50 ; \mathrm{H}, 5.85$. Found: C, 50.45; H, 5.93 .

$( \pm)-1$ '-(2,3,4,6-Tetra- $O$-acetyl- $\beta$-D-glucopyranosyl)-4'-(2-O-acetylpropyl)-1H-1',2',3'-triazole (4b)

Solid; mp 117-119 ${ }^{\circ} \mathrm{C}$; [a] ${ }_{\mathrm{D}}^{25}=-13.2\left(\mathrm{C}=0.007 / \mathrm{CH}_{2} \mathrm{CL}_{2}\right) ;{ }^{1} \mathrm{H}$ NMR $(400$ $\left.\mathrm{MHz}, \mathrm{CDCl}_{3}\right): \delta 7.59\left(\mathrm{~s}, 1 \mathrm{H}, \mathrm{H}_{\text {triaz }}\right), 5.85(\mathrm{dd}, 1 \mathrm{H}, J 9.0 \mathrm{~Hz}, \mathrm{H}-1), 5.45-5.22(\mathrm{~m}$, $3 \mathrm{H}, \mathrm{H}-2, \mathrm{H}-3, \mathrm{H}-4), 5.15(\mathrm{~m}, 1 \mathrm{H}, \mathrm{CHO}), 4.32$ (dd, $1 \mathrm{H}, J 12.0,4.7 \mathrm{~Hz}, \mathrm{H}-6 \mathrm{a})$, 4.16 (dd, 1H, $J 12.0,2.0 \mathrm{~Hz}, \mathrm{H}-6 \mathrm{~b}$ ), 4.00 (ddd, $1 \mathrm{H}, J 10.2,4.7,2.0 \mathrm{~Hz}, \mathrm{H}-5$ ), 2.96-2.89 (m, $\left.2 \mathrm{H}, \mathrm{CH}_{2} \mathrm{O}\right), 2.09$ (s, $\left.3 \mathrm{H}, \mathrm{CH}_{3} \mathrm{CO}\right), 2.07$ (s, 3H, $\left.\mathrm{CH}_{3} \mathrm{CO}\right), 2.03(\mathrm{~s}$, $\left.3 \mathrm{H}, \mathrm{CH}_{3} \mathrm{CO}\right), 2.06\left(\mathrm{~s}, 3 \mathrm{H}, \mathrm{CH}_{3} \mathrm{CO}\right), 1.87\left(2 \mathrm{~s}, 3 \mathrm{H}, \mathrm{CH}_{3} \mathrm{CO}, \mathrm{R}\right.$ and $\left.\mathrm{S}\right), 1.28-1.25$ $\left(2 \mathrm{~d}, 3 \mathrm{H}, J 6.3,6.3 \mathrm{~Hz}, \mathrm{CH}_{3}, \mathrm{R}\right.$ and $\left.\mathrm{S}\right) .{ }^{13} \mathrm{C} \mathrm{NMR}\left(100 \mathrm{MHz}, \mathrm{CDCl}_{3}, \mathrm{R}\right.$ and $\left.\mathrm{S}\right): \delta$ $170.4(2 \mathrm{C}), 169.8,169.4,168.9,144.5,120.1,86.0$ and $85.8,75.2$ and $75.0,73.1$ and 72.6, 70.2, 69.8 and 68.5, 67.7 and 67.6, 61.5, 32.1, 21.2 (2C, R and S), 20.7, 20.5 (2C, R and S), 20.5, 20.1 and 20.0 (R and S), 19.6 and 19.4 (R and S). Anal. Calc.: $\mathrm{C}_{21} \mathrm{H}_{29} \mathrm{~N}_{3} \mathrm{O}_{11}$ : C, 50.50; H, 5.85. Found: C, 50.62; H, 5.83.

$( \pm)-1$ '-(2,3,4,6-Tetra- $O$-acetyl- $\beta$-D-glucopyranosyl)-4'-(1-O-acetylethyl)-1 $H-1$ ', 2',3'-triazole (4c)

Solid; mp $164-166{ }^{\circ} \mathrm{C}$; [a] ${ }^{25}=-35.3\left(\mathrm{C}=0.002 / \mathrm{CH}_{2} \mathrm{CL}_{2}\right) ;{ }^{1} \mathrm{H}$ NMR $(400$ $\left.\mathrm{MHz}, \mathrm{CDCl}_{3}\right): \delta 7.79\left(\mathrm{~s}, 1 \mathrm{H}, \mathrm{H}_{\text {triaz }}\right), 6.05(\mathrm{~m}, 1 \mathrm{H}, \mathrm{CHO}), 5.87(2 \mathrm{~d}, \mathrm{R}$ and $\mathrm{S}, 1 \mathrm{H}$, $J 8.6 \mathrm{~Hz}, \mathrm{H}-1), 5.46-5.22(\mathrm{~m}, 3 \mathrm{H}, \mathrm{H}-2, \mathrm{H}-3, \mathrm{H}-4), 4.32(\mathrm{dd}, 1 \mathrm{H}, J 12.7,5.1 \mathrm{~Hz}$, H-6), 4.16 (br d, $1 \mathrm{H}, J 12.7 \mathrm{~Hz}, \mathrm{H}-6$ ) , 4.01 (ddd, $1 \mathrm{H}, J 10.2,4.7,2.0 \mathrm{~Hz}, \mathrm{H}-5$ ), $2.10\left(\mathrm{~s}, 3 \mathrm{H}, \mathrm{CH}_{3} \mathrm{CO}\right), 2.08\left(\mathrm{~s}, 3 \mathrm{H}, \mathrm{CH}_{3} \mathrm{CO}\right), 2.07\left(\mathrm{~s}, 3 \mathrm{H}, \mathrm{CH}_{3} \mathrm{CO}\right), 2.03(\mathrm{~s}, 3 \mathrm{H}$, $\left.\mathrm{CH}_{3} \mathrm{CO}\right), 1.87\left(\mathrm{~s}, 3 \mathrm{H}, \mathrm{CH}_{3} \mathrm{CO}\right), 1.68\left(\mathrm{~d}, 3 \mathrm{H}, J 5.5 \mathrm{~Hz}, \mathrm{CH}_{3}\right) .{ }^{13} \mathrm{C} \mathrm{NMR}(100 \mathrm{MHz}$, $\mathrm{CDCl}_{3}, \mathrm{R}$ and S): $\delta 170.5,170.2,169.9,169.3,168.7,146.0,106.9,85.8,75.2$, 72.7 and $72.6,70.3$ and $70.2,67.7,64.8$ and $64.7,61.5,29.7,21.2(2 \mathrm{C}, \mathrm{R}$ and S), 20.7, 20.5, 20.4, 20.1, 19.6 and 19.3 (R and S). Anal. Calc.: $\mathrm{C}_{20} \mathrm{H}_{27} \mathrm{~N}_{3} \mathrm{O}_{11}: \mathrm{C}$, 49.48; H, 5.61. Found: C, 49.25; H, 5.40.

1 '-(2,3,4,6-Tetra- $O$-acetyl- $\beta$-D-glucopyranosyl)-4'-(1-O-acetylcyclohexyl)-1H-1',2',3'-triazole (4d)

Solid; mp 148-150 ${ }^{\circ} \mathrm{C}$; [a] ${ }_{\mathrm{D}}^{25}=-7.0\left(\mathrm{C}=0.001 / \mathrm{CH}_{2} \mathrm{CL}_{2}\right) ;{ }^{1} \mathrm{H}$ NMR $(400$ $\left.\mathrm{MHz}, \mathrm{CDCl}_{3}\right): 7.72\left(\mathrm{~s}, 1 \mathrm{H}, \mathrm{H}_{\text {triaz }}\right),{ }_{5.86}\left(\mathrm{~d}, 1 \mathrm{H}, J 8.6 \mathrm{~Hz}, \mathrm{H}^{-1}\right), 5.48-5.22(\mathrm{~m}, 3 \mathrm{H}$, H-2, H-3, H-4), 4.32 (dd, $1 \mathrm{H}, J 4.7,12.5 \mathrm{~Hz}, \mathrm{H}-6 \mathrm{a}$ ), 4.15 (br d, $1 \mathrm{H}, J 12.5 \mathrm{~Hz}$, H-6b), $4.02(\mathrm{~m}, 1 \mathrm{H}, \mathrm{H}-5), 2.40-2.36\left(\mathrm{~m}, 4 \mathrm{H}, 2 \mathrm{CH}_{2}\right.$, cyclohexyl), $2.03(\mathrm{~s}, 3 \mathrm{H}$, $\left.\mathrm{CH}_{3} \mathrm{CO}\right), 2.00\left(\mathrm{~s}, 3 \mathrm{H}, \mathrm{CH}_{3} \mathrm{CO}\right), 1.96\left(\mathrm{~s}, 3 \mathrm{H}_{1} \mathrm{CH}_{3} \mathrm{CO}\right), 1.92\left(\mathrm{~s}, 3 \mathrm{H}, \mathrm{CH}_{3} \mathrm{CO}\right), 1.81$ (s, 3H, $\left.\mathrm{CH}_{3} \mathrm{CO}\right), 1.79-1.32$ (m, 6H, cyclohexyl). ${ }^{13} \mathrm{C}$ NMR (100 $\left.\mathrm{MHz}, \mathrm{CDCl}_{3}\right)$ : $\delta 170.5,169.9(2 \mathrm{C}), 169.3,168.9,157.1,120.3,85.6,76.5,75.2,72.7,67.8$, 61.6, 35.7, 34.7, 29.7, 25.2, 22.0, 22.0, 21.8, 20.5, 20.0, 18.2, 16.6. Anal. Calc.: $\mathrm{C}_{24} \mathrm{H}_{33} \mathrm{~N}_{3} \mathrm{O}_{11}: \mathrm{C}, 53.43 ; \mathrm{H}, 6.17$. Found: C, 53.53; H, 6.20.

2-[4-(O-acetyl-methyl)-1H-1,2,3-triazol-1-yl)]-1,4-naphtoquinone (6)

Solid; mp 150-152 ${ }^{\circ} \mathrm{C} ;{ }^{1} \mathrm{H}$ NMR $\left(400 \mathrm{MHz}, \mathrm{CDCl}_{3}\right): \delta 8.70\left(\mathrm{~s}, 1 \mathrm{H}, \mathrm{H}_{\mathrm{H}}\right)$, 8.23-8.20 (m, $\left.2 \mathrm{H}, \mathrm{H}_{\text {napht }}\right), 7.86-7.84\left(\mathrm{~m}, 2 \mathrm{H}, \mathrm{H}_{\text {nanhth }}\right), 7.75\left(\mathrm{~s}, 1 \mathrm{H}, \mathrm{H}_{\text {nanhth }}\right), 5.31$ $\left(\mathrm{s}, 2 \mathrm{H}, \mathrm{CH}_{2}\right), 2.11(\mathrm{~m}, 3 \mathrm{H}, \mathrm{OAc}) .{ }^{13} \mathrm{C}$ NMR $\left(100 \mathrm{MHz}, \mathrm{CDCl}_{3}\right): \delta 183,178.5$, 170.1, 143.3, 138.5, 134.4, 133.8, 130.8, 126.6, 126.2, 125.9, 125.4, 56.6, 20.2. Anal. Calc.: $\mathrm{C}_{15} \mathrm{H}_{11} \mathrm{~N}_{3} \mathrm{O}_{4} \times 0.3 \mathrm{H}_{2} \mathrm{O}: \mathrm{C}, 59.51 ; \mathrm{H}, 3.87 ; \mathrm{N}, 13.88$. Found: C, 59.70; H, 3.55 .
2-[4-(1-O-acetyl-1-methyl-ethyl)-1 H-1,2,3-triazol-1-yl)]-1,4naphtoquinone (7)

Solid; mp 165-166 ${ }^{\circ} \mathrm{C}$; ${ }^{1} \mathrm{H}$ NMR $\left(400 \mathrm{MHz}, \mathrm{CDCl}_{3}\right): \delta 8.61\left(\mathrm{~s}, 1 \mathrm{H}, \mathrm{H}_{\text {triaz }}\right)$, 8.21-8.15(m, $\left.2 \mathrm{H}, \mathrm{H}_{\mathrm{moth}}\right), 7.87-7.84\left(\mathrm{~m}, 2 \mathrm{H}, \mathrm{H}_{\mathrm{manh}}\right), 7.77\left(\mathrm{~s}, 1 \mathrm{H}, \mathrm{H}_{\mathrm{rath}}\right), 2.03$ (s, $3 \mathrm{H}, \mathrm{OAc}), 1.92\left(\mathrm{~s}, 6 \mathrm{H}, \mathrm{CH}_{3}\right) .{ }^{13} \mathrm{C} \mathrm{NMR}\left(100 \mathrm{MHz}, \mathrm{CDCl}_{3}\right): \delta 183.8,179.3$, $170,152.6,139.3,135,134.4,131.4,127.2,126.5,123.2,76.3,27.3,22.2$. Anal. Calc.: $\mathrm{C}_{17} \mathrm{H}_{15} \mathrm{~N}_{3} \mathrm{O}_{4} \times 0.3 \mathrm{H}_{2} \mathrm{O}: \mathrm{C}, 61.72 ; \mathrm{H}, 4.77$. Found: $\mathrm{C}, 61.72 ; \mathrm{H}, 4.33$.

2-[4-(prop-1-en-2-yl)-1H-1,2,3-triazol-1-yl)]-1,4-naphtoquinone (8) Solid; mp 137-139 ${ }^{\circ} \mathrm{C}$; ${ }^{1} \mathrm{H}$ NMR (400 MHz, $\left.\mathrm{CDCl}_{3}\right): \delta 8.53\left(\mathrm{~s}, 1 \mathrm{H}, \mathrm{H}_{\mathrm{nanth}}\right)$ 8.16-8.09 (m, 2H, $\left.\mathrm{H}_{\text {naphth }}\right), 7.81-7.76\left(\mathrm{~m}, 2 \mathrm{H}, \mathrm{H}_{\text {naptht }}\right), 7.71\left(\mathrm{~s}, 1 \mathrm{H}, \mathrm{H}_{\text {triaz }}\right), 5.85$ $(\mathrm{s}, 1 \mathrm{H}, \mathrm{C}=\mathrm{CH}) 5.16(\mathrm{~s}, 1 \mathrm{H}, \mathrm{C}=\mathrm{CH}), 2.13\left(\mathrm{~s}, 3 \mathrm{H}, \mathrm{CH}_{3}\right) .{ }^{13} \mathrm{C} \mathrm{NMR}(100 \mathrm{MHz}$, $\left.\mathrm{CDCl}_{3}\right): \delta 183.1,178.9,149.1,138.6,134.4,133.7,131.9,130.9,126.6,125.9$, 125.5, 120.8, 113.5, 19.9. Anal. Calc.: $\mathrm{C}_{15} \mathrm{H}_{11} \mathrm{~N}_{3} \mathrm{O}_{2}: \mathrm{C}, 67.92 ; \mathrm{H}, 4.18$. Found: C, 67.88; H, 4.33 .

\section{RESULTS AND DISCUSSION}

Protection and deprotection of hydroxyl groups in chemistry has been a tool commonly used in carbohydrate chemistry. To begin our studies we chose the carbohydrate D-glucose for acetylation (Scheme 1). First, we used mont $\mathrm{K} 10$ and $\mathrm{Ac}_{2} \mathrm{O}$ in an ultrasound bath in the absence of a solvent. We differed the amount of mont. K10 from 20 to $200 \%$ (w/w), acetic anhydride (1 to 4 equiv/OH), and reaction time (15 or $30 \mathrm{~min}$ ). Then, was determined the best condition, i.e., mont. $200 \%, 4$ equiv of $\mathrm{Ac}_{2} \mathrm{O}$ per hydroxyl group, and $15 \mathrm{~min}$ under ultrasound irradiation at room temperature.

After that, was exchanged mont. K10 to KSF and the yields of reactions were very close, $92 \%$ and $94 \%$, respectively. The stereoselectivity at the anomeric carbon showed a ratio $\alpha: \beta$ of $3: 1$ with KSF, while for K10 the ratio was 3:2. We also obtained best results when montmorillonite K10 without activation was tested, which reacted with a complete conversion for acetylated products. However, when clay was used after the activation process, the reaction was not complete. Apparently, the presence of water inside the cagelike structure or channels in the clay enhanced the acoustic cavitations in this reaction. Another aspect this reaction is the difference between anhydride and monohydrated D-glucose, which has the best results for anhydride glucose, since it was necessary to add 20 equiv $\mathrm{Ac}_{2} \mathrm{O}$ more to complete the reaction when monohydrated D-glucose was used. In this case, probably molecular water hydrolyzes the anhydride and consequently causes loss of their reactivity. Nevertheless, in regard to stereoselectivity, significant difference was not found.
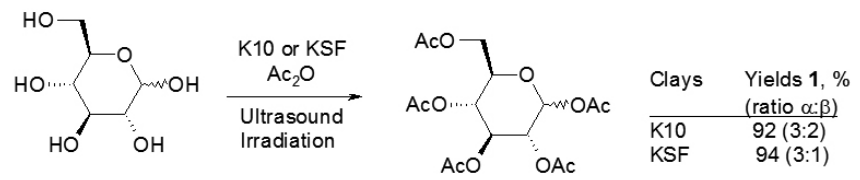

D-glucose

1

Scheme 1. Acetylation of D-glucose using US and clay conditions

In order to analyze the reusability of the K10 and KSF clays, the catalyst was recovered at the end of the reaction and its recycling use was evaluated immediately after the first reaction. The solid acid was removed by filtration and washed with ethyl acetate. The catalyst lost its activity after four reuses $(<$ $70 \%$, see Fig. 1). Each time, the recovered catalyst was found to be reusable and showed a range of yields from $92 \%$ to $50 \%$ after seven catalytic cycles using K10. The results for KSF were close, as shown in Figure 1.

To continue our studies, we selected mont. K10 clay as the standard catalyst for our acetylation procedure. We focused our attention on molecules such as $N$-glucosyl triazolic sugars, glycerol, D-mannitol and some 2-substituted 1,4-naftoquinone structures.

For the synthesis of $N$-glucosyl triazolic alcohols, we started from the per-O-acetilated glucose $\mathbf{1}$ via azide sugar $\mathbf{2}$, using the click triazole synthesis methodology based on ultrasonic energy developed in our laboratory. ${ }^{13}$ Then, the triazolic sugars 3a-d were obtained in 10-40 min with yields between 60 and $98 \%$ (Scheme 2, Table 1).

The acetylation step, using our optimized protocol $\left(\mathrm{Ac}_{2} \mathrm{O} / \mathrm{K} 10 / \mathrm{US} / \mathrm{rt}\right)$, produced acetylated compounds 4a-d in moderate to good yields of 47-73 $\%$ (Scheme 2, Table 1). The $N$-glucopyranoside was compatible with these reaction conditions without hydrolysis. Only in one case the starting material was recovered (Entry 2, Table 1). In all cases, no elimination products were detected. 


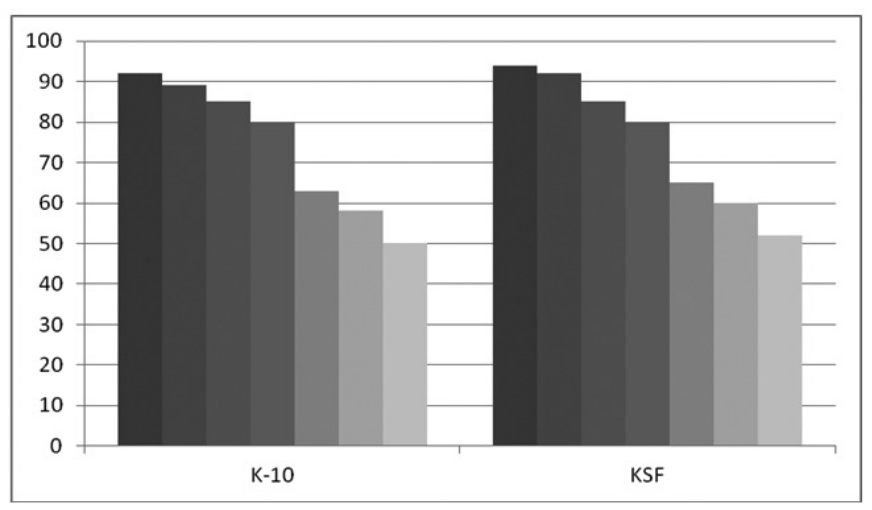

Figure 1. Reusability of K10 and KSF from D-glucose acetylation reaction
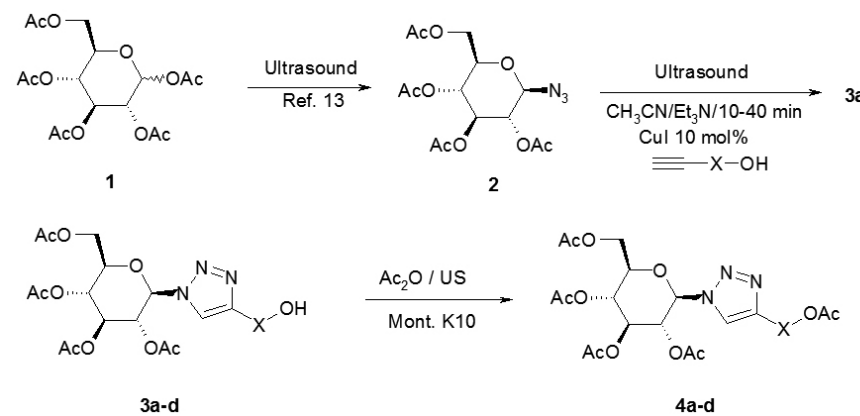

Scheme 2. Synthesis of $N$-glucosyl triazolic 3a-d and 4a-d using US and clay conditions

Table 1. Synthesis of the compounds 3a-d and $\mathbf{4 a - d}$ using US and clay conditions.

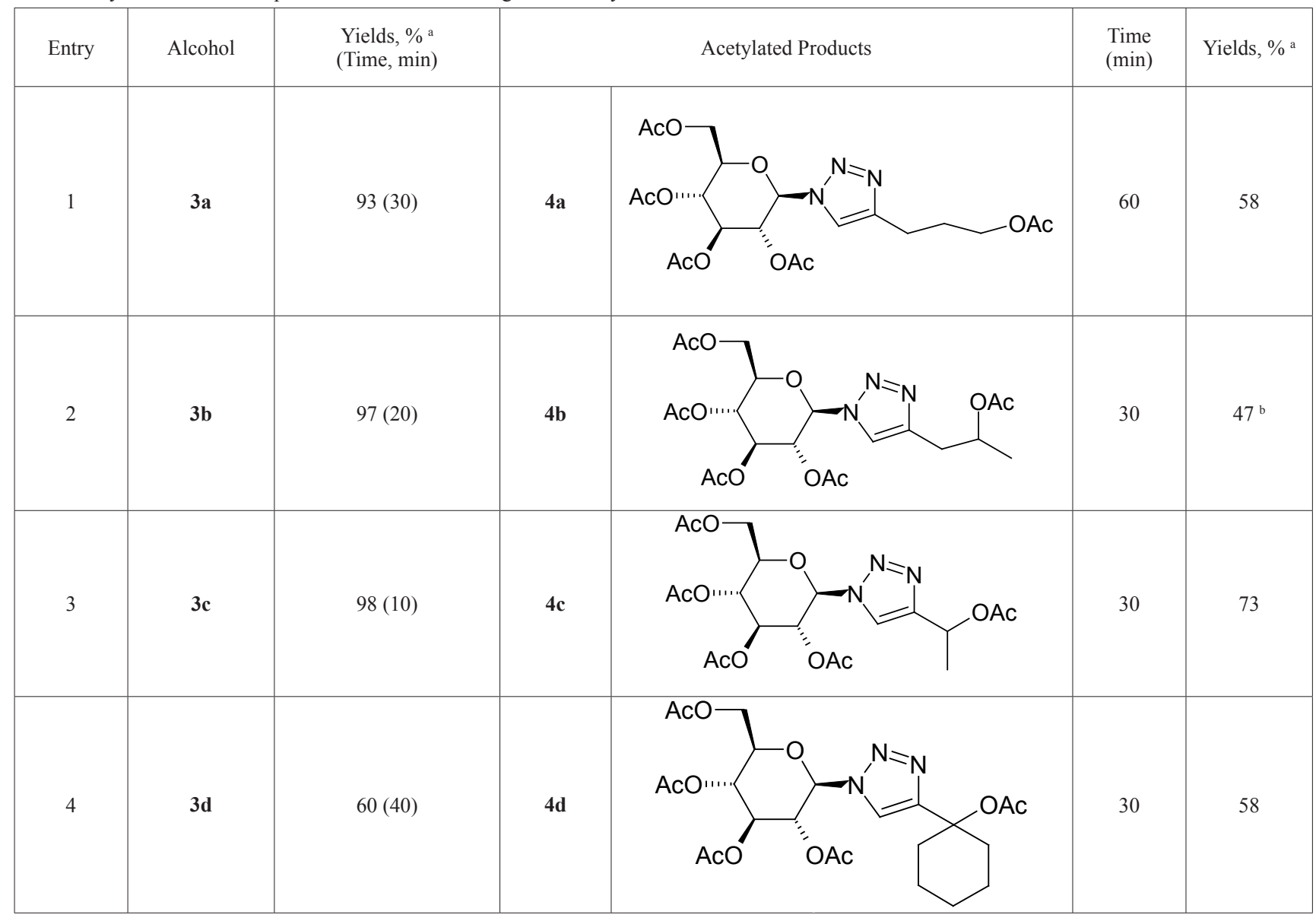

${ }^{\text {a }}$ After column chromatography; ${ }^{\text {b }}$ Recovered starting materials.

To prepare derivates containing the 1,4-naphtoquinone moiety, we started from 2-[4-hydroxymethyl-1 $H$-1,2,3-triazol-1-yl)-1,4-naphthoquinone 5a and 2-[4-(2-hydroxy1-2-methyl-ethyl-)-1 $H$-1,2,3-triazol-1-yl]-1,4-naphthoquinone $\mathbf{5 b}$, that were synthesized using a method recently described by us. ${ }^{18}$ The acetylation reaction was performed at $50{ }^{\circ} \mathrm{C}$ during $40 \mathrm{~min}$ with some drops of dichloromethane added to facilitate solubility. The acetylated compound $\mathbf{6}$ was obtained in yields of $77 \%$ after column chromatography (Scheme 3). At room temperature, the results were similar; however, the reaction time takes 60 $\min$. From the compound $\mathbf{5 b}$, after a reaction time of 40 min, the product 7 was obtained in $58 \%$, and the by-product 8 was isolated in yields of 37\% as a vinyl derivative due to their dehydration. Normally, these alkenyl type compounds may be prepared from dehydration of tertiary alcohol linked to a 1,2,3-triazole function using $\mathrm{POCl}_{3} / \mathrm{HCl}^{19}$ Additionally, this mild condition was not efficient in promoting acetylation of 2-amino-1,4-naphthoquinone, which is expected for this kind of of extensive conjugated molecule, which requires harsher conditions $(\mathrm{RCOCl} / \mathrm{NaH}){ }^{20}$ 


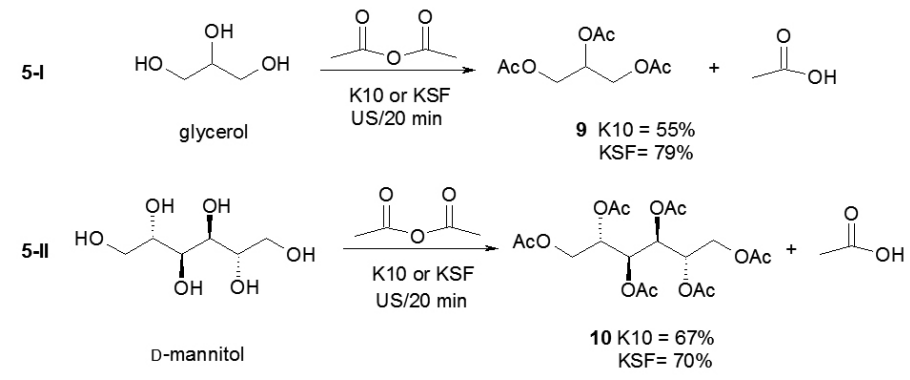

Scheme 3. Synthesis of 2-substituted 1,4-naftoquinones 6-8 using US and clay conditions

After that, we tried the exclusive preparation of $\mathbf{8}$; however, without success from $\mathbf{5 b}$ using acid conditions $\left(\mathrm{HCl}, \mathrm{H}_{2} \mathrm{SO}_{4}, \mathrm{AcOH}\right)$. On the other hand, when we employed $\mathrm{AcOH} /$ reflux from acetylated compound 7, the compound $\mathbf{8}$ was formed (Scheme 3). Then, we conclude that by-product 8 only could be obtained from 7. We have found in the literature results that can explain this effect. ${ }^{21}$ Delatouche and co-workers ${ }^{21}$ describes mildly acid conditions to furnish amine derivatives and conjugated alkene from triazolylcarbamates as leaving group. Based on this information we proposed an elimination mechanism. First, a complex between K10 and carbonyl group was formed, which after triazole protonation at $\mathrm{N}^{3}$ the conjugated base $\left(\mathrm{AcO}^{-}\right)$may be remove a proton at methyl position to form $\mathbf{8}$ and acetic acid (Scheme 4), an assisted version of a classical E2 mechanism.

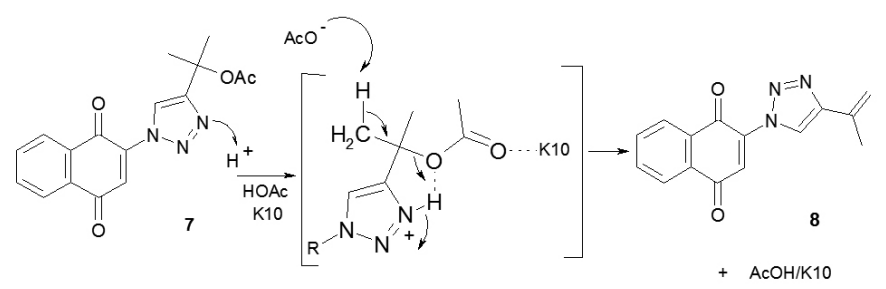

Scheme 4. Proposed elimination mechanism for the formation of alkene 8

In the next step, we investigated the preparation of triacetil derivative 9 (Scheme 5-I). This compound is able to be produced in an acid-catalyzed reaction of glycerol with acetic anhydride. ${ }^{22}$ The tri-acetylglycerol 9 was synthesized in yields of $55 \%$ employing montmorillonite K10 as the catalyst. On the other hand, when KSF was employed, the yield was higher at $79 \%$. This result may be rationalized according to literature that describes KSF as more efficient than $\mathrm{K} 10$ because of its higher acidic character. ${ }^{23}$

$$
\text { D-I }
$$

Scheme 5. Synthesis of tri-acetylglycerol 9 and hexa- $O$-acetyl-D-mannitol 10 using US and clay conditions

The literature describes the use of EI-MS $(70 \mathrm{eV})$ for analysis of triacetylated glycerol showing the peaks $m / z 218,145\left(\mathrm{M}-\mathrm{CH}_{2} \mathrm{OAc}\right), 103$ $(100 \%)$ and 43 as main ions. ${ }^{24}$ In our study, the analysis of the CG-MS showed fragmentation expected for 9 (Scheme 6). Nevertheless, our CG-MS spectrum did not show the molecular ion $[\mathrm{M}]^{+}$. We observed the peak at $159(\mathrm{M}-\mathrm{AcOH})$ with $100 \%$ relative abundance, as shown in Figure 2. To explained the ion $\mathrm{m} / \mathrm{z} 159$, we are suggesting that after the molecular ion $[\mathrm{M}]^{+}$is formed, there immediately occurs a $1,2-H$ rearrangement to obtain the structure A (Scheme 5). From structure A we proposed three pathways for fragmentation. Firstly, a possible nucleophilic attack from a carbonyl group to eliminate acetic acid and forming the cyclic structure B (pathway-a). Another pathway is the loss of radical AcO $\times$ leading to structure $\mathrm{B}^{\prime} \mathrm{m} / \mathrm{z} 159$ (pathway-b). The third possibility from structure $\mathrm{A}$ is the elimination of the radical $\mathrm{AcOCH}_{2} \times$ to form structure C $m / z 145$ (pathway-c). To rationalize the peak $m / z 103$ (structure D), we assumed initially an intramolecular hydrogen transfer and then loss of ketene $\left(\mathrm{O}=\mathrm{C}=\mathrm{CH}_{2}\right)$. The acylium ion $\mathrm{m} / \mathrm{z} 43$ was formed mainly from $[\mathrm{M}]^{+}$after loss of the radical $\mathrm{AcOCH}_{2} \mathrm{CH}(\mathrm{OAc}) \mathrm{CH}_{2} \mathrm{O} \times$. We also proposed fragmentation from ion $\mathrm{m} / \mathrm{z} 103$ to form $\mathrm{m} / \mathrm{z} 43$ and ethylene diol, as depicted in Scheme 6 .

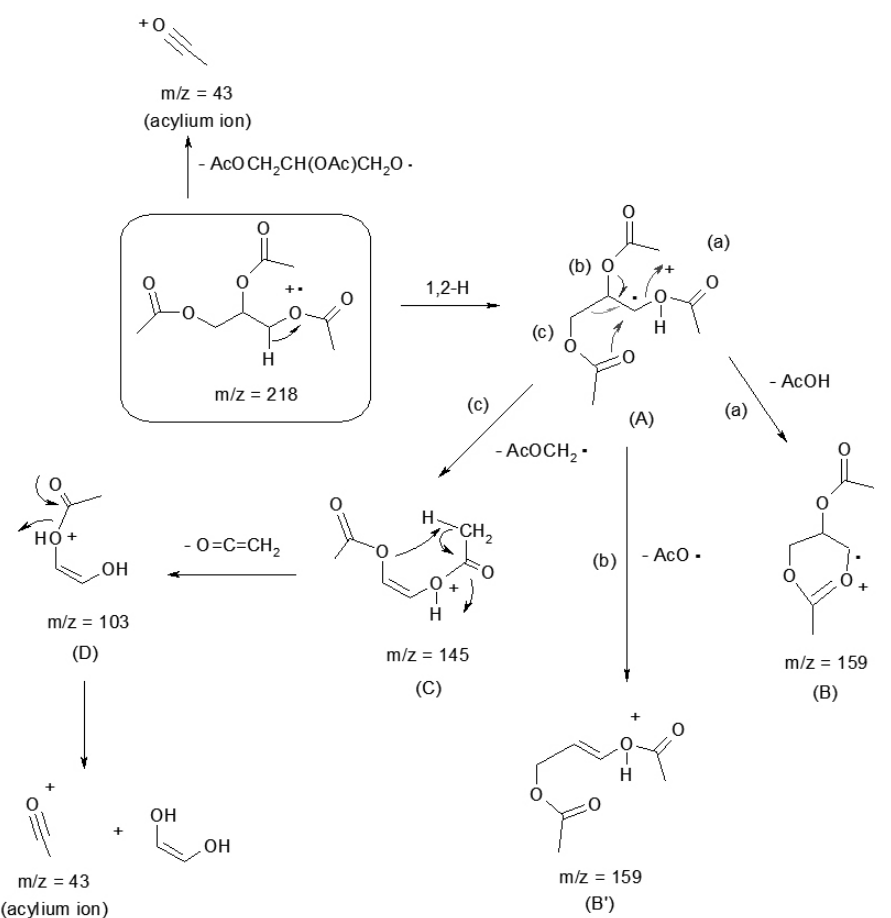

Scheme 6. Proposed fragmentation for tri-acetylglycerol 9

Hexa-O-acetyl-D-mannitol $\mathbf{1 0}$ has received attention recently from the scientific community. ${ }^{25,26} \mathrm{We}$ acetylated D-mannitol using K10 or KSF to furnish hexa- $O$-acetyl-D-mannitol $\mathbf{1 0}$ in yields of $67 \%$ and $70 \%$, respectively (Scheme 5-II). The ${ }^{1} \mathrm{H}$ NMR analysis showed the proton shift and coupling constant expected for this compound.

\section{CONCLUSION}

In this work, we revisited a versatile methodology for the acetylation reaction of important molecules that have a diversity of applications. For this purpose, we have combined clays (montmorillonite K10 or KSF) and ultrasound energy to explore the acetylation reaction on natural or synthetic compounds such as D-glucose, D-mannitol, glycerol and 1,2,3-triazolic derivatives, i.e., $N$-glucopyranosyl sugars and 2-substutated 1,4-naftoquinoid structures. The acetylated products were obtained in moderate to good yields. This is a faster, eco-friendly and remarkable procedure for prompt use in the formation of acetyl derivatives of alcohols in laboratory.

\section{ACKNOWLEDGEMENTS}

The authors are grateful to FACEPE-PRONEM (1232.1.06/10) for financial support. W. S. Nascimento and P. L. F. Costa are gratefully to FACEPE and V. N. e Melo to CAPES for providing a fellowship. Our thanks are also due to Analytical Centers CENAPESQ-UFRPE for facilities installation and DQFUFPE for obtaining the spectral data.

\section{REFERENCES}

1. D. Enders, A. A. Narine, J. Org. Chem. 73, 7857, (2008)

2. N. Iranpoor, H. Firouzabadi, E. E. Davan, Tetrahedron Lett. 54, 1813, (2013) 
3. S. Gibson, D. Romero, H. K. Jacobs, A. S. Gopalan, Tetrahedron Lett. 51, 6737, (2010)

4. J. W. J. Bosco, A. Agrahari, A. K. Saikia, Tetrahedron Lett. 47, 4065, (2006)

5. E. Kattnig, M. Albert, Org. Lett. 6, 945, (2004)

6. K. Shimizu, T. Higuchi, E. Takasugi, T. Hatamachi, T. Kodama, A. Satsuma, J. Mol. Catal. A: Chem. 284, 89, (2008)

7. A. X. Li, T. S. Li, T. H. Ding, Chem. Commun. 1389, (1997)

8. V. R. Choudhary, K. Y. Patil, S. K. Jana, J. Chem. Sci. 116, 175, (2004)

9. T. Cseri, S. Békássy, Z. Bódás, B. Ágai, F. Figueras, Tetrahedron Lett. 37, 1473 (1996)

10. B. M. Choudary, M. Sateesh, M. L. Kantam, K. V. S. Ranganath, K. V. Raghavan, Catal. Lett. 76, 231 (2001)

11. T. J. Mason, J. P. Lorimer, Applied Sonochemistry: Uses of Power Ultrasound in Chemistry and Processing. Wiley-VCH, Verlag $\mathrm{GmbH}$ : Weinheim, Germany, 2002.

12. C. J. P. Barros, J. J. R. de Freitas, R. N. de Oliveira, J. R. de Freitas Filho, J. Chil. Chem. Soc. 56, 721 (2011)

13. G. B. Silva, B. M. Guimarães, S. P. O. Assis, V. L. M. Lima, R. N. de Oliveira, J. Braz. Chem. Soc. 24, 914 (2013)

14. M. T. da Silva, R. N. de Oliveira, W. O. Valença, F. C. G. Barbosa, M. G. da Silva, C. A. Camara, J. Braz. Chem. Soc. 23, 1839 (2012)

15. (a) S. Brochette, G. Descotes, A. Bouchu, Y. Queneau, N. Monnier, C.
Pétrier, J. Mol. Catal. A: Chem. 123, 123 (1997); (b) G. D. Yadav, M. S. M. M. Rahuman, Ultrason. Sonochem. 10, 135 (2003)

16. B. Sreedhar, R. Arundhathi, M. A. Reddy, G. Parthasarathy, Appl. Clay Sci. 43, 425 (2009)

17. (a) A. L. Xavier, Monograph. Uso de Argila e Energia de Ultrassom na Reação de Acetilação de Carboidratos. Federal Rural University of Pernambuco (2010); (b) A. L. Xavier, R. N. de Oliveira, III Workhsop SO Norte, Nordeste e Centro-Oeste. March (2010), Maceió-AL.

18. W. S. Nascimento, C. A. Camara, R. N. de Oliveira, Synthesis 20, 3220 (2011)

19. R. J. Thibault, K. Takizawa, P. Lowenheilm, B. Helms, J. L. Mynar, J. M. J. Fréchet, C. J. Hawker, J. Am. Chem. Soc. 128, 12084 (2006)

20. J. C. Lien, L. J. Huang, J. P. Wang, C. M. Teng, K. H. Lee, Chem. Pharm. Bull. 44, 1181 (1996)

21. R. Delatouche, M. Mondon, A. Gil, G. Frapper, C. Bachmann, P. Bertrand, Tetrahedron 67, 401 (2011)

22. L. N. Silva, V. L. C. Gonçalves, C. J. A. Mota, Catal. Commun. 11, 1036 (2010)

23. D. Habibi, O. Marvi, Arkivoc xiii, 8 (2006)

24. B. Nobel, M. Mittelbach, G. Uray, Anal. Chem. 80, 8712 (2008)

25. J. R. Satam, R. V. Jayaram, Catal. Commun. 9, 2365 (2008)

26. A. Kamal, M. N. A. Khan, K. S. Reddy, Y. V. V. Srikanth, T. Krishnaj, Tetrahedron Lett. 48, 3813 (2007) 